\title{
A Preliminary Study on the Vitality Ordering of Minority Languages in China
}

\author{
Sun Hongkai *
}

\begin{abstract}
Ordering the minority languages in China in terms of their vitality can help decide what priorities are in securing the most endangered ethnic languages. In view of the actual condition of the Chinese ethnic languages, this paper proposes some corresponding comprehensive indexes and ranks over 100 ethnic languages of China in terms of their vitality.
\end{abstract}

Key words: minority languages in China, endangered languages, language vitality, ordering

\section{Introduction}

At the opening of the 21st century, the vitality of many less privileged languages is declining. With the globalization of the world's economy and the accelearation of regional economic development, with the popularization of such media as radio broadcasting, television, and internet commnication, with the rapid opening of isolated and semi-isolated regions and ethnic groups, and with the increased dissemination of official languages and lingua francas, this decline appears inevitable, despite human goodwill to the contrary. Therefore, the issue of endangered languages has become a hot topic in the realm of ethnic minority language studies, and also a hot topic of the international linguistic community. As a medium of human cultures, the loss of languages will result in the disappearance of some important human cultural phenomena, which is a great loss of human intangible wealth. Linguists and ethnic minority groups, as well as experts on intangible human cultures and government administrators are all worried about this looming disastrous prospect.

In recent years, this issue of endangered languages has also aroused the attention of China's central leadership, since cultural diversity figures as an important content of establishing a socialist harmonious society. Languages are the medium of cultures; as the

\footnotetext{
* Sun Hongkai: Institute of Ethnology and Anthropology, Chinese Academy of Social Sciences, Beijing 100081, China. Contact address: No. 1205, Building 26, Anding Road, Beijing 100029, China. E-mail: hksun@public3.bta.net.cn.
} 
old Chinese saying goes, "With the skin gone, what can the hair adhere to?" To put it plainly, cultures cannot exist without languages as their medium. Their decay and extinction will pose serious challenges to cultural diversity. For this reason, the Central Government of China has launched the Preservation Programme for China's Ethnic and Civil Cultures (the Preservation Programme hereafter), for which the Ministry of Culture is responsible. In 2004, Zhou Heping, the deputy-minister of the Ministry of Culture and director of the Preservation Programme, declared: "the Preservation Programme for China's Ethnic and Civil Cultures is a large-scale, wide-scope, and systematic project that is organized, promoted and implemented by the Central Government. Its purpose is the efficient preservation of precious, endangered ethnic and civil cultures, which are of historic, cultural and scientific value. It is mindful of past experiences in the preservation of ethnic and civil cultures and mindful also of new situations and new characteristics of the new era. This programme is planned to come to completion in seventeen years, and will involve systematic planning and steady implementation of the preservation of China's ethnic and civil cultures, with an endeavour to provide basic scientific, rational, planned and orderly preservation of China's ethnic and civil cultures" (Zhou Heping 2004).

The general objective of the Preservation Programme is as follows: "By 2020, the precious and endangered ethnic and civil cultures of historic, cultural and scientific values will undergo efficient preservation; a preliminary comprehensive preservation institution and system will be established; awareness of protecting the ethnic and civil cultures will be aroused among the whole community and throughout the society; scientific, standardized and computerized preservation will basically be fulfilled. In order to accomplish this objective, the Protection Programme will be carried out in three phases: the first phase is from 2004 to 2008, which will involve the pilot preservation and rescuing of the endangered cultures; the second phase will begin from 2009 and end in 2013, which will revolve around the overall unfolding of the programmes and concentrate on protecting the key ones; from 2014 to 2020 will be the third phase, which will be a period for the improvement and perfection of the mechanisms."

The objects of the Preservation Programme mainly constitute the ethnic and civil traditional cultures which are of historic, cultural and scientific value, including specifically:

(1) traditional oral literature as well as languages and scripts;

(2) traditional operas, Chinese folk art forms (including ballad singing, story telling, comic dialogues, clapper or cross talks, etc.), music, dancing, fine arts and acrobatics; 
(3) traditional artifacts, arts and workmanships;

(4) traditional rituals, festivals, festivities and sports activities;

(5) typical original data, artifacts and localities related to the first four items mentioned above.

Languages are of top priority to be preserved, which shows their importance and the urgency to protect them.

\section{Why endangered languages should be protected}

It is universally held that languages are an important medium for human beings to communicate and to exchange ideas. As far as their function as a communication tool is concerned, the fewer the languages in the world, the more convenient it ought to be to communicate and exchange ideas. Suppose there were only one single language in the world, how convenient it would be for people to talk with each other.

However, languages are products of history. Languages have originated and developed along with the shaping and development of various nations; hence, languages have contained records of the rising and falling of different nations. Besides the function of communication and exchange of ideas, languages still retain other functions. Let us take the following examples to illustrate.

First of all, as the medium of cultures, languages have in their stock and preservation rich cultural phenomena. Various literary products such as stories, myths, legends, fables, poetry, lyrics, riddles, and operas and dramas, which have been composed with and retained in languages, are definitely expressed through languages. The epics recovered from the diverse ethnic minority languages of China are rich and popular, and they not only have exerted tremendous impact on the society but also enriched China's cultural treasury. To cite just a few examples, consider the following: Gasar of the Tibetans, the Jiangger of the Mongolians, the Manas of Kerkiz, Ashima of the Yis, Genesis of the Naxis, The Bainiaoyi of the Zhuangs, the Qinniangmei of the Kam Ethnic Group, the Denghua of the Hmongs as well as Lebaozhaiwa of the Jingpos. These are only a miniscule fraction of the literature of the ethnic minorities. It is not an exaggeration to say that almost all ethnic minority groups who still retain their mother tongues possess their own literature. The oral literary pieces, which have been recovered, sorted out and made available to the readers, account for only an extremely minor part of the whole treasure.

Secondly, as a medium of information, a language represents the overall knowledge and experience accumulated over the past hundreds and thousands of years by its native 
speakers. This includes the accumulation of their knowledge and experience fighting against nature, and the historical experience of their steady survival by living in an organized community. Languages have been indispensable to the achievement of all these, and to their transmission from generation to generation, as well as to their recording and dissemination across and beyond space and time. In this sense, all human knowledge and experience are preserved and able to be disseminated through languages.

Thirdly, as a cognitive system and a direct manifestation of thinking processes, languages embody native speakers' cognitive and perceptual system for organizing the natural world around them. There are universals to this system but also peculiarities. This system steadily becomes increasingly precise with the evolution of the human community and human society. Hence, the danger of permanently losing both a language and the ways of thinking that it represents is a matter of great concern to humanity. Last but not least, as a significant identity of a nation or an ethnic group, a language distinguishes one nation or group from all the others. An ethnic group attaches special feelings to their native tongue. Consequently, the loss of languages will generally impose an unbearable shadow on the psychology of a given ethnic group.

As for linguistics in its own right, each language constitutes a resource that is precious to the development of linguistics. It is evident that the richer the linguistic resources accumulate are, the greater the potential will be for the development of linguistics. To take China as an example, there are a large number of lesser known languages in China. Up till now, no in-depth investigation has been conducted on them. Though these languages do not boast a large body of speakers, they are invaluable academically. Many of them have retained countless ancient features of Sino-Tibetan and Altaic language families. Certain linguistic resources and data, through in-depth research, will prove to be keys to numerous historical riddles. Therefore, implementing the rescuing documentation and preservation of the extremely endangered and almost extinct language data is an urgent vital task of developing the study of linguistics in China, as well as one fundamental work of the study of ethnic minority languages. It will promote and enhance the research of descriptive linguistics, comparative linguistics, typological linguistics and even ethnic paleography. In addition, it will benefit the in-depth research into the historical relations between and among different ethnic groups of China, enhance the understanding of the complexity of China as an integrated nation of diverse distinct ethnic groups, help improve the work on ethnic groups, and help promote the unification and progress of China's ethnic groups. 
To sum up, languages are important treasures of human beings. The extinction of endangered languages is a general tendency of the globe under the new situation of the new era and is a serious challenge to linguistic and cultural diversity. It is an extremely urgent task for a state composed of divergent ethnic groups and possessing numerous languages to attach importance to endangered languages. It is also a holy vocation for a conscientious linguist to attach enough importance to endangered languages, which they should always involve themselves in.

\section{The basis for language vitality ordering}

As languages are important media of cultures as well as the major objects of ethnic and civil cultural protection in China, protecting language diversity should be of top priority among our future tasks. There are more than 130 languages (not including various dialects) in China; thus we should work out an ordering in terms of their degrees of vitality and rescue the most endangered ones with regard to their degree of endangerment and urgency.

However, ascertaining the facts of the degrees of language vitality requires a systematic project. What criteria to be used in order to objectively order the languages in China according to their degrees of vitality is very difficult to establish. On the 10th to 12th of March, 2003, UNESCO organized and hosted in its Paris headquarters an international expert conference on endangered languages, and nearly one hundred delegates from over forty countries as well as from various funds of endangered languages were present.

During the conference, the UNESCO Ad Hoc Expert Group on Endangered Languages presented to the conference their draft documents, entitled Language Vitality and Endangerment. The document reviewed some important activities on endangered languages by the UNESCO, emphasized the importance of the issue of endangered languages, analysed the causes of language endangerment, and discussed how to identify endangered languages; more importantly, it proposed which measures be taken on protecting endangered languages.

At present, the document by the UNESCO Ad Hoc Expert Group on Endangered Languages has been made public through the Internet. In regard to how to assess language vitality, nine evaluative factors have been proposed, which can be regarded as the

\footnotetext{
(1) The present author, on behalf of the Chinese Academy of Social Sciences (CASS), attended the conference, presented our views on endangered languages and participated in the discussion and decision as regards language vitality, language endangerment as well as action and plan proposals.
} 
guidelines concerning the study of the vitality of ethnic minority languages in China. These are as follows:

Factor 1: Intergenerational language transmission

Factor 2: Absolute number of native language speakers

Factor 3: Proportion of speakers within the total population

Factor 4: Shifts in domains of language use

Factor 5: Response to new domains and media

Factor 6: Materials for language education and literacy

Factor 7: Governmental and institutional language attitudes and policies, including official status and use

Factor 8: Community members' attitudes toward their own language

Factor 9: Amount and quality of documentation

Based on the above assessment factors, language vitality are divided into six ranks in the expert group's document from dynamic to inactive, which can be summarised as in the following: the expert group determined that languages' vitality may be ranked on a 6-point scale from dynamic to inactive, as follows:

(1) dynamic

(2) robust/ active

(3) dwindling vitality, threatened and showing some signs of endangerment

(4) coping vitality, definitely endangered

(5) minimal vitality, severely endangered

(6) inactive, critically endangered or extinct

According the use and distribution of the ethnic minority languages in China, three more factors can be added as assessment factors to the nine ones mentioned above. They are:

(1) distribution of language community, i.e., concentrated, scattered or living together with other ethnic groups

(2) degree of internal variation of the given language, i.e., the more varied the language is, the lower the vitality

(3) distribution of languages transnationally, that is, across national borders.

In addition, other factors such as the development of transportation networksand economic development of the community will also exert some influence on language vitality, which should also be taken into consideration.

\section{The vitality ordering of minority languages in China}


There are more than 130 ethnic minority languages in China, belonging respectively to five language families, viz. Sino-Tibetan, Altaic, Austronesian, Austroasiatic, and Indo-European, among which the first two rank first in terms of the number of affiliated languages, whereas the third and fourth rank second. The ethnic minority languages in China can be classified into six scales according to the evaluative factors presented above.

\subsection{Dynamic languages}

The manifestations are as follows: the proportion of monolingual speakers is high; no problem exists as to the intergenerational transmission of mother tongues; the absolute number of speakers is comparatively large; language planning is promoted by the institutions at the level of autonomous regions or autonomous prefectures; an established orthography is available, and a large number of publications are also available; there is radio broadcasting in the mother tongues; the languages are spoken both at home, in the markets and in the schools and in the administration and legislature; the awareness of maintaining the mother tongues is strong; there are large areas of concentrated distribution of the language community; although there is dialectal variation, the orthography is available to serve as a means of unifying the dialects. Languages of this type consist mostly of Uighur, Tibetan, Korean, Mongolian, Kazak, Zhuang and Yi.

\subsection{Robust/ active languages}

They manifest the following features: the proportion of monolingual speakers is relatively high; intergenerational language transmission is not at all threatened; the absolute number of speakers is large; language policy is promoted by organizations at the level of autonomous regions or autonomous prefectures, but of lesser intensity; the written forms of the languages are available and boasts a fairly large number of publications; there is radio broadcasting in the mother tongues, but of lower frequencies and the effect is not so desirable; the mother tongues are used not only at home, in the markets and in schools but sometimes also in administrative and legislative bodies; the mother tongue speakers are aware of mother tongue maintenance; there are fairly large areas of concentrated distribution; in some cases of this type of languages, dialectal variation is small, or in some other cases, even though there is some dialectal variation, the existing writing serves as a unifying force; or in still other cases, the language boasts neither a large number of speakers nor an available writing, yet as a compensating factor, there is a high proportion of monolingual speakers as the language community has very little contact with the outside because it is hard to get to. This language type includes such languages as Lisu, Dai, 
Hmong (Miao), Li, Hani, Kam (Dong), Sui (Shui), Durong (Dulong), Buyei (Buyi), Yamei (Dawu), Lahu and Xibe.

\subsection{Languages of dwindling vitality, threatened and showing some signs of} endangerment

The facts are: the proportion of monolingual speakers is comparatively low, while most are bilingual or multilingual speakers; intergenerational mother tongue transmission is threatened in some areas of the language community; the absolute number of speakers is decreasing; language planning is weakly implemented or suffers from lack of administration; no writing is available, or in the case where there are written forms, few publications are available in the writing; there is no radio broadcasting in native tongues, or even if there is such broadcasting, it has hardly more than a formal existence, with no virtual effect; languages are used only inside families, scarcely or not used at all in the markets or at schools, not to mention in administration or legislature; the awareness of mother tongue maintenance is weak, and in some cases, even if the speech community is aware, they can do nothing to revert the tendency and the worsening situation; the distribution is relatively scattered; the dialectal variation is large and there is low mutual intelligibility, and thus communication cannot be conducted in the mother tongues. Qiang, De'ang, Daur, Naxi, rGyarong, Tajik, Jingpo, Zaiwa, Tujia, Molao, Dongxiang, Bao'an, Bulang, Bai, Salar, Ergong, Lingao, Mien, Amei, and Maonan are languages belonging to this type.

\subsection{Languages of coping vitality and definitely endangered}

The major manifestations of this type are as follows: there are few monolingual speakers or no such speakers at all, mostly bilingual or multilingual speakers; intergenerational language transmission is interrupted or broken, and the majority of speakers are middle-age and senior members of a particular language community, that is, the youngest speakers are of the parental generation; the absolute number of speakers is drastically decreasing; there is no administration of language planning; basically no writing in the language is available; no radio broadcasting is conducted in the native language; the mother tongue is spoken only at home or of restricted use inside the hamlets or villages, scarcely or not used at all in the markets or at schools, let alone used in the administrative or legislative bodies; the awareness of mother tongue maintenance is weak, or even if they have the awareness, they have no way out to stop the worsening situation; the community lives in a scattered manner, encompassed or surrounded by communities of dominant languages; dialectal variation is large, thus no communication can be conducted in the mother tongue due to the low intelligibility among dialects. Languages belonging to this type mostly include Gelao, 
Purmi (Pumi), Jinuo, Nusu, Menba, Yido, Cangluo, Jing, Langsu, Leqi, Lawurong, Gemen, Darang, Yugur, Eronqin (Elunchun), Uzbek, Ersu, Namuyi, Muya, Guiqiong, Shixing, Zhaba, Queyu, Cun, Huihui, Biao, Laji, Bugeng, Russian, Hu, Jiongnai, Lakkia, Bunu, Baheng, Boga'er, Rukai, Zou, Bunong, Beinan, Paiwan, Kemu, Bana, Tanglang, Mang, Kazhuo, Ewenqi, Kirgiz, Chadong, Sangkong, Bisu, Mo, Yanghuang, Baima, and Mo'ang.

\subsection{Languages of minimal vitality and severely endangered}

The major manifestations are: there are no monolingual speakers, that is, all are bilingual or multilingual, and they tend to be more proficient in their second languages than in their mother tongues; intergenerational language transmission critically affected, and the language is spoken only by the grandparental generation and up, whereas those younger all shift to using other languages; the absolute number of speakers is low; there is no language planning administration; no written forms for the mother tongues exist; no radio broadcasting is available in the mother tongues; the mother tongue is spoken only at home, ceasing to be used at all in other communication contexts such as in the markets or at schools, let alone in the administrative or legislative bodies; the speakers basically lack the awareness of mother tongue maintenance, or in some cases, even if they have the awareness, they have no way out to reverse or stop the worsening situation; the community is distributed in a scattered manner, encompassed or surrounded by dominant languages; dialectal variation is big, no communication can be conducted in the mother tongue due to the low intelligibility among dialects; the structures of mother tongues have been deteriorated, and no narrators of oral literature are available, with the lexicon drastically decreasing. Languages belonging to this type mostly include Anong, Hezhen, Tatar, She, Pubiao, Lai, Kangjia, Rouruo, Tuva, Xiandao, Pola, Kamalan, Tayal, Sadaq, Kemie, Saixia, Buxing, Sulong, Bongru.

\subsection{Inactive, critically endangered or extinct languages}

Languages of this type have utterly ceased to function in any communication context and social domain, and their major manifestations are: there are absolutely no monolingual speakers, and most members of the language communities have shifted to using other languages; what is worse, there are no mother tongue speakers at all: the languages leave some traces only in the memories of some individual senior members or in old documents; only some individuals know about the mother tongues, which have ceased to be employed as media of communicating or thinking. This type consists of Manchu, Mulao, Hakas, Yi, Kanakanavu, Sha'alu'a, Basehai and Shao. 
Sun Hongkai

\section{Related discussions}

Among the ethnic minority groups of China, the majority speak their mother tongues, but in some cases, two or more languages are simultaneously spoken by some ethnic groups, while on the other hand, some members of some ethnic groups or a minority of the community members use other languages as their first language. Therefore, the number of languages actually spoken is greater than the number of the ethnic groups. For example, among the Gaoshan ethnic group in Taiwan, at least 15 Austronesian languages are spoken (1). The Yao ethnic group in South China uses six languages, namely, Mien, Bunu, Lakkia, Baheng, Jiongnai, and Younuo. Members of the Luoba ethnic group speak at least four languages, respectively Bogar, Yido, Sulong and Bengru. Three languages are spoken by the Nu ethnic group, which are Nusu, Rouruo and Anong. The Jingpo ethnic group speaks Zaiwa, Leqi, Bola, Langsu and Xiandao as well, along with the Jingpo language. For more details concerning issues in this regard, please refer to my article On the scientific Recognition of Languages in China (Application of Languages and Writings, No. 3, 2005) as well as On the Recognition of Languages in China, the second chapter of Part I in Languages in China, with the present author as chief editor (the Commercial Press, 2006). Thus, the language names in this paper sometimes are identical with those of the ethnic groups, and sometimes not.

Language vitality is a dynamic process, as the language domain and degree of use of every and each language are under constant change; consequently it cannot be precisely defined in a static manner. The evaluative factors presented above are based on experiences drawn from the investigation, documentation and study of endangered languages through the past decade by the expert group of UNESCO, and proposed and decided after continuous discussion conducted at international conferences. Among these factors, some are universally applicable, but some others are not totally applicable to the situations in China. As a result, we only regard the factors as guidelines for reference when we work out the ordering of language vitality in China, which relies heavily on the actual national situation in China. In the past two decades, the present author has been heavily involved in the investigation of the ethnic minority language use situation in China. On the basis of experience and knowledge from the investigations as well as the data accumulated in those prolonged efforts, I have worked out the preliminary vitality ordering of all these ethnic

\footnotetext{
(1) In regard to the language use situation in Taiwan, besides the information that we have long had, Professor Doctor Jackson Sun Tian-sin, deputy director of the Language Institute of the Academia Sinica, has also provided us with much new information; in addition, Ms Chen Shujuan from Xinzhu Teachers' College has also provided a great deal of new information. Special thanks go to both of them.
} 
minority languages in China, referring at the same time to Study on the Vitality of Ethnic Minority Languages in China (Press of China Central University for Nationality Studies, 2000), a research result by Professor Huang Xing. Hence unavoidably and common to all such studies, this ordering is to some extent subjective, and the results may be in variation from the actual situation.

In addition, although we classify all the minority languages in China into six vitality types abiding by the evaluative factors figured out by the expert group, the languages in each group still bear their own features and vary in different ways from each other; therefore the ordering is only an attempt to provide a point of reference.

China is undertaking the Conservation Programme of Ethnic and Civil Cultures (the Conservation Programme). As an object of this Programme, endangered languages have already been on the agenda: pilot studies have been undertaken on two such languages (Yugur and Eronqin). We sincerely hope that the result of this paper can be of some help to the future work of the Programme. At least, this can give us some idea of what are the most important and urgent cases, to put it more clearly, which languages are critically endangered, and hence should be on the list of top priority to be investigated, documented and rescued among the very first ones; otherwise, they will disappear entirely before any timely documentation and rescuing action is taken, which will cause irreversible and irremediable losses to the whole mankind.

Extinction and disappearance is an unavoidable and irreversible natural tendency. Therefore, what we can do presently is to preserve the materials of endangered languages, or to slow down the speed of decline and extinction of endangered languages. As to the maintenance of endangered languages in order that they may not be become abandoned or extinct, or to reverse its endangerment, this is an issue that presents more difficulty. As far as the present situation is concerned, the preservation of the materials of an endangered language should at least involve the following:

(1) Document the lexicon of the given language in large quantity, with no less than 10,000 entries, with an endeavor to compile a medium-sized dictionary. On the basis of these, figure out the phonological system of the language.

(2) Document a great number of the sentences of the language, and work out the grammatical system of the language: the more detailed, the better.

(3) Notate in International Phonetic Alphabet all kinds of traditional oral literature and texts, including stories, ballads and folk songs, fables, and poetic epics or genesis, and 
arrange them in interlinear forms of four lines if possible (to be specific, writing in native orthography, phonetic symbols, literal translation and free translation).

(4) Document the dialect and subdialects of the language, including not only the material of a certain dialect center, but also the panoramic scenario of the whole language and its dialects, subdialects and vernacular speech varieties, that is, the combination of both the in-depth descriptions of particular spoken varieties of the language and the global description of the overall language.

The devices of documentation should be all dimensional and all-round, comprehensive and multi-dimensional. Besides the literary materials, the materials documented should include the corresponding audio-visual materials, digital forms being the best. After sorting out and compilation, these recordings and audio-visual materials as well as literary documents will need to be kept in language museums and serve as reference for future research and reconstruction or revitalisation.

Establish a harmonious language community, that is, in a community of divergent language varieties, all members, besides using their mother tongues, can acquire fluency at the same time in a lingua franca-like speech of that particular community, and more importantly, the vitality of their mother tongues will not be affected or interrupted: this is an ideal language use and co-existence situation, which calls for the joint efforts of all involved, ranging from linguists to government administrators, members of all ethnic groups as well as members of the whole community.

\section{References}

Huang Xing. 2000. On the Vitality of Minority Languages in China. [M] China Central University for Nationality Studies Press.

Kazuto Matsumura Ed. 1998. Studies in Endangered Languages. [Z] Paper from the International Symposium on Endangered Languages. Tokyo. November 18-20, 1995.

Lenore A. Grenoble and Lindsay J. Whaley Ed.. 1998. Endangered Languages. [M] Current issues and future prospects. Cambridge University Press.

Sun Hongkai. 1999a. A Survey into the Unknown Languages in China. [A] In Shi Feng \& Pan Wuyun (eds): New Frontiers of Chinese Linguistics. [C] Hong Kong City University Press.

Sun Hongkai. 1999b. The Anon Language: a Follow-up Observation on the Increasing Seriously Endangered Language. [J] Zhongguo Yuwen. No. 5.

Sun Hongkai. 2001a. On the Endangered Languages. [J] Language Teaching and Research. No. 1.

Sun Hongkai. 2001b. Bilingualism and Endangered Language Protection. [A] In Wang Yuanxin (ed): Bilingual Teaching and Research. Vol.3. [C] China Central University for Nationality Studies Press.

Sun Hongkai. Ed. 1997-2005. The Series of Newly Discovered Languages in China. [M] Shanghai Yuandong Publishing House. China Central University for Nationality Studies Press and Minzu Press.

Sun Hongkai, Hu Zengyi and Huang Xing Ed.. 2006. Languages in China. [M] The Commercial Press.

The Institute of Ethnology and Anthropology of the Chinese Academy of Social Sciences and the National Commission for Ethnic Affairs Ed. 1994. On the Language Use of Minority Languages in China. [M] Tibetology Press. 


\section{A Preliminary Study on the Vitality Ordering of Minority Languages in China}

UNESCO Ad Hoc Expert Group on Endangered Languages. 2003. Language Vitality and Endangerment. [Z] Document submitted to the International Expert Meeting on UNESCO Programme Safeguarding of Endangered Languages. Paris. March 10-12, 2003.

Xu Shixuan. 2001. On the Endangered Languages. [M] China Central University for Nationality Studies Press.

Zhou Heping. 2004. The Conservation Programme of China's Ethnic and Civil Cultures. [Z] In the Publicizing Materials compiled by State Administration Centre for The Conservation Programme of China's Ethnic and Civil Cultures. 